University of New Hampshire

University of New Hampshire Scholars' Repository

7-1992

\title{
Differential Phase Estimation with the SeaMARC II Bathymetric Sidescan Sonar System
}

\author{
M A. Masnadi-Shirazi \\ Shiraz University \\ Christian de Moustier \\ University of California - San Diego \\ Pierre Cervenka \\ University of California - San Diego \\ Stanley H. Zisk \\ University of Hawaii at Manoa
}

Follow this and additional works at: https://scholars.unh.edu/ccom

Part of the Oceanography and Atmospheric Sciences and Meteorology Commons

\section{Recommended Citation}

Masnadi-Shirazi, M.A.; de Moustier, C.; Cervenka, P.; Zisk, S.H., "Differential phase estimation with the SeaMARCII bathymetric sidescan sonar system," in Oceanic Engineering, IEEE Journal of , vol.17, no.3, pp.239-251, Jul 1992 doi: 10.1109/48.153441

This Journal Article is brought to you for free and open access by the Center for Coastal and Ocean Mapping at University of New Hampshire Scholars' Repository. It has been accepted for inclusion in Center for Coastal and Ocean Mapping by an authorized administrator of University of New Hampshire Scholars' Repository. For more information, please contact Scholarly.Communication@unh.edu. 


\section{Differential Phase Estimation with the SeaMARC II Bathymetric Sidescan Sonar System}

Mohammed A. Masnadi-Shirazi, Member, IEEE, Christian de Moustier, Member, IEEE, Pierre Cervenka, and Stanley H. Zisk

\begin{abstract}
A maximum likelihood estimator is used to extract differential phase measurements from noisy seafloor echoes received at pairs of transducers mounted on either side of the SeaMARC II bathymetric sidescan sonar system. Carrier frequencies for each side are about $1 \mathrm{kHz}$ apart, and echoes from a transmitted pulse, 2 ms. long, are analyzed. For each side, phase difference sequences are derived from the full complex data consisting of base-banded and digitized quadrature components of the received echoes. With less bias and a lower variance, this method is shown to be more efficient than a uniform mean estimator. It also does not exhibit the angular or time ambiguities commonly found in the histogram method currently used in the SeaMARC II system. A figure for the estimation uncertainty of the phase difference is presented and results are obtained for both real and simulated data. Based on this error estimate and an empirical verification derived through coherent ping stacking, a single filter length of $\mathbf{1 0 0} \mathbf{~ m s}$ is chosen for data processing applications.
\end{abstract}

\section{INTRODUCTTON}

$I^{\mathrm{N}}$ N RECENT years, swath bathymetric sidescan sonar systems have been used efficiently in seafloor profiling. The principle of operation is based on determination of the acoustic angle of an echo from the seabed by measurement of the electrical phase difference produced by the echo received at two rows of transducer arrays. Knowing the acoustic angle and the slant range, or time of arrival of seafloor echoes at the arrays, one can simply calculate the depth and the horizontal distance of the seabed reflector. The SeaMARC II (Sea Mapping and Remote Characterization) system, operated by the Hawaii Institute of Geophysics, is a seafloor mapping system that works on this phase measurement principle [1], [2].

A common problem with all the phase measuring sidescan sonar systems, including SeaMARC II, is that their phase measurements are dispersed by noise and interferences. The main sources of interference are multiple

Manuscript received April 28, 1992. This work was supported by the Office of Naval Research under Grants N00014-91-J-1073 and N00014$90-\mathrm{J}-1106$. Part of this paper was presented at the 120th Meeting of the Acoustical Society of America, November 1990.

M. A. Masnadi-Shirazi is with the Department of Electrical Engineering, Shiraz University, Shiraz, Iran.

C. deMoustier and P. Cervenka are with the Marine Physical Laboratory, Scripps Institute of Oceanography, LaJolla CA 92093-0205.

S. H. Zisk is with the School of Ocean and Earth Science and Technology, University of Hawaii, Manoa, 96822.

IEEE Log Number 9201763. reflections on the ocean surface and bottom, as well as interferences due to multiple targets on the bottom at the same range. A theoretical noise estimation method and details about the sources of interference are discussed in [1]. The first step in bathymetry processing is to estimate the correct phase difference from the measured phase data. To this end various statistical methods, such as mean angle and mean time methods and histogram approaches, have already been applied and tested [1], [2]. In the mean angle approach, the uniform mean of the phase difference over fixed time intervals is obtained, so that small sample groups of data are represented instead of all the instantaneous phase differences. In the mean time approach the uniform mean of time samples is taken over fixed angle intervals. The histogram method consists in binning the differential phase data in a 2-d histogram of phase versus time and selecting a modal time for every angle bin, or alternatively, a modal angle for every time bin.

Results of the histogram method show that the phase sample distributions in time or in angle are not symmetric [1]. The asymmetry in the distribution in time is caused by the fact that as the travel time increases the spread of the differential phase data increases due to progressively higher transmission loss and lower signal to noise ratio (SNR). The asymmetry in the phase sample distribution in angle, although not as significant as for time, is well explained by the $2 \pi$ ambiguity in differential phase measurements [3]. Thus, in both cases the mean value does not follow the mode of the histogram and hence the mean is biased. As a result, uniform mean estimation methods of phase are not accurate and the histogram methods have been preferred over the mean methods.

The drawbacks of the histogram method are a more cumbersome processing compared to the mean methods, and ambiguities introduced by modal points having the same time for different angle bins, or the same angle for different time bins. Note that ambiguities resulting form echoes arriving at the same time from different directions are inherent to the sidescan geometry. Likewise, this geometry requires that phase angles vary monotonically with increasing time. For simple 2-row systems, such ambiguities cannot be resolved regardless of the phase processing method. However, ambiguities arising from noisy phase values have the same deleterious effect in a his- 
togram processing because all the values have the same weight.

In this paper we introduce a different approach which works with the full complex received signal, that has been base-banded and quadrature sampled, with subsequent application of a maximum likelihood estimator to obtain the phase difference. The corresponding theory is briefly reviewed in Section II, and its application to SeaMARC II data is presented in Section III. An analytical model and computer simulation for the SeaMARC II System is performed in Section IV to make the study and analysis of the system more flexible and easier. This model is then used to study the effect of the transmitted signal frequency bandwidth, filter length, and noise level on the estimation uncertainty results.

\section{THEORY OF OPERATION}

\section{A. Sample Recording}

The principle of operation of a swath bathymetry sidescan sonar is illustrated in Fig. 1 where one side of the system is represented. The system must have at least two rows of transducers (e.g., A and B in Fig.1) whose acoustic centers are a distance $d$ apart. Typically, one or both of these rows are used to transmit a gated pulse of the carrier frequency over a single fan beam whose maximum response axis has been oriented to provide as much coverage on the seafloor as possible. For a pulse $T_{0} \mathrm{~s}$ long, the transmitted signal taken a distance $r$ from the array has the form

$S_{t}(t)= \begin{cases}E(t) \cos \left(\omega_{c} t+k r\right), & \text { when } \frac{r}{c}<t<\frac{r}{c}+T_{0} \\ 0, & \text { elsewhere }\end{cases}$

where $E(t)$ is the amplitude, $\omega_{c}$ is the carrier angular velocity, $k=2 \pi / \lambda$ is the wave number ( $\lambda$ being the acoustic wavelength), $r=c t$ is the one-way slant range, and $c$ is the sound velocity in water. The initial phase is arbitrarily set to zero.

The backscattered bottom echoes are received independently by each row, so that it is possible to measure the electrical phase difference of the signals received at row $A$ versus those received at row $B$. The signal received at row A from a single scatterer, $S_{r A}(t)$ has a form similar to (1), with magnitudes $M_{A}(t)$ and phases $\phi_{A}(t)$ :

$S_{r A}(t)=M_{A}(t) \cos \left(\omega_{c} t+\phi_{A}(t)\right) \quad$ with $\phi_{A}(t)=k r_{A}$.

Likewise, the signal received at row $\mathrm{B}$ from a single scatterer, $S_{r B}(t)$ is

$S_{r B}(t)=M_{B}(t) \cos \left(\omega_{c} t+\phi_{B}(t)\right) \quad$ with $\phi_{B}(t)=k r_{B}$.

$r_{A}$ and $r_{B}$ are the instantaneous round trip slant ranges to rows $\mathrm{A}$ and $\mathrm{B}$ for a given reflector on the seafloor. As expected, the phase difference $\Delta \phi(t)=\phi_{A}(t)-\phi_{B}(t)$ is simply related to the distance traveled by the backscat-

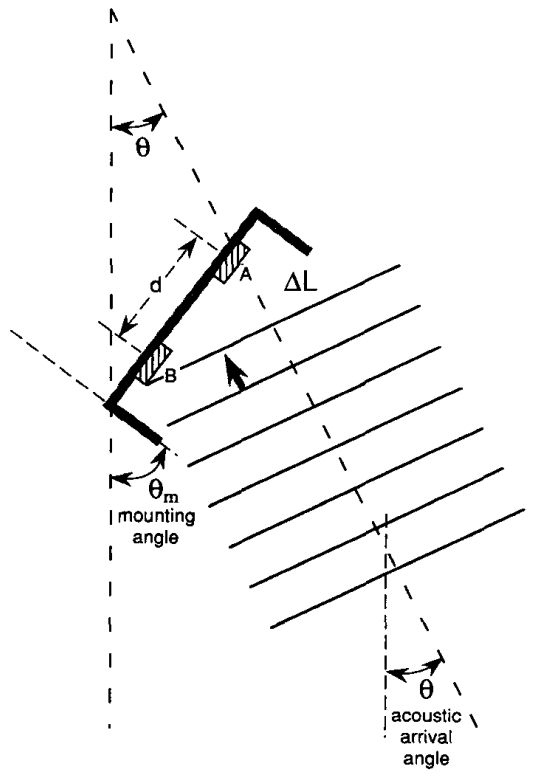

Fig. 1. Acoustic arrival angle measuring geometry.

tered wave front from one row to the other. From the geometry of Fig. 1, the relationship between this phase difference and the angle of arrival $\theta$ of the backscattered signal is then

$$
\Delta \phi(t)=\frac{2 \pi d}{\lambda} \sin \left(\theta_{m}-\theta(t)\right)
$$

$\theta_{m}$ is the array mounting angle and $\theta$ is the angle of arrival of the echo with respect to vertical down.

Given that phase can only be measured to $2 \pi$, (4) indicates that $\Delta \phi(t)$ and $\theta$ are unambiguously related provided $d \leq \lambda / 2$. This equation represents the ideal case: perfect beam patterns, single scatterers, coherent signals, no noise. In practice, the scattered signal is the summation of many elementary signals produced from single stochastic scatterers distributed in the ocean volume or on the seafloor. System noise is also added to the signal at the receiver. Thus, the received signal is a stochastic noisy process and the phase difference measurement is affected by noise and interferences, and one must resort to an estimation process. If the stochastic process is narrow-band, it can be represented by (2) and (3), $M(t)$ and $\phi(t)$ being the envelope and the phase of the process, and $\omega_{c}$ the central frequency of its spectrum. In addition, the continuous representations given in (1)-(4) need to be considered in a discrete sense as one usually deals with digitized samples of these functions.

Assuming a quadrature receiver as shown in Fig. 2, the base-banded output of the digitizer consists of the quadrature components of the signal, e.g. for row A:

$$
I_{A}(n)=M_{A}(n) \cos \phi_{A}(n)
$$




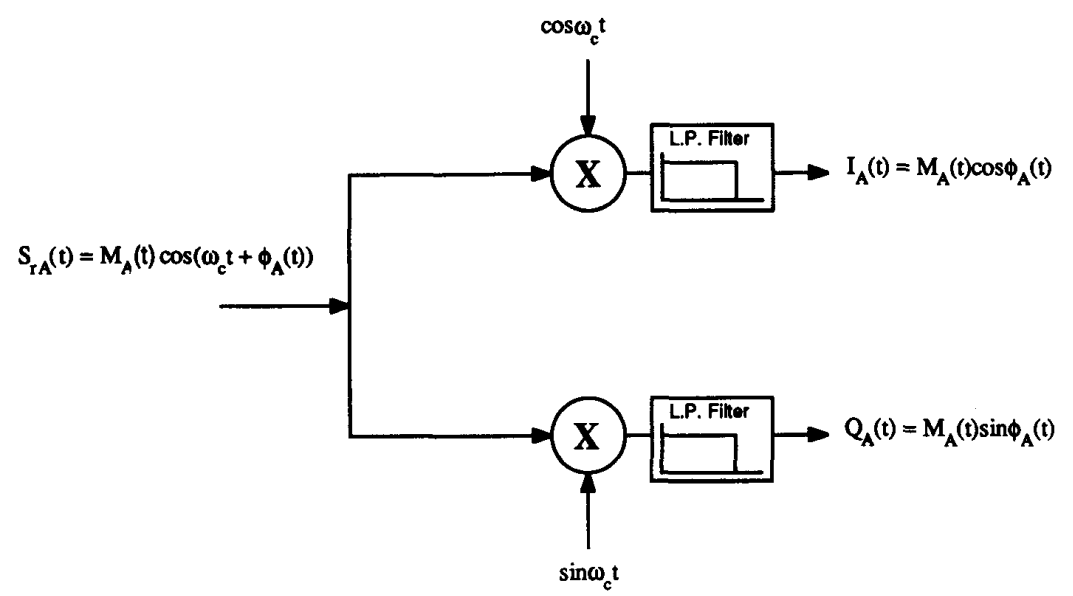

Fig. 2. Quadrature receiver block diagram.

and

$$
Q_{A}(n)=M_{A}(n) \sin \phi_{A}(n) .
$$

The phase difference $\Delta \phi(n)$ is therefore easily computed from the individual phases:

$\Delta \phi(n)=\tan ^{-1}\left\{Q_{A}(n) / I_{A}(n)\right\}-\tan ^{-1}\left\{Q_{B}(n) / I_{B}(n)\right\}$

Although this approach is straightforward, the noisy nature of the data makes it difficult to derive reliable differential phase estimates from (7) [1], [2].

\section{B. Differential Phase Computation and Smoothing Process}

Here, instead of only looking at the phase term, our approach deals with the complete signal. To this end, the signal sequences $S(n)$ are expressed in complex notation for analytical convenience. We have for row A:

$$
S_{A}(n)=M_{A}(n) e^{j \phi_{A}(n)} \text {. }
$$

Likewise, for row $B$,

$$
S_{B}(n)=M_{B}(n) e^{j \phi_{B}(n)} .
$$

The real parts of (8) and (9) correspond to the in-phase (e.g., (5)) components of the signal and the imaginary parts to the quadrature components (e.g., (6)).

Multiplying (8) by the complex conjugate of (9) yields

$\vec{P}(n)=S_{A}(n) S_{B}^{*}(n)=M_{A}(n) M_{B}(n) e^{j\left(\phi_{A}(n)-\phi_{B}(n)\right)}$.

$\vec{P}(n)$ is a vector in the Fresnel plane and represents a complex stochastic sequence whose phase, $\Delta \phi(n)=$ $\phi_{A}(n)-\phi_{B}(n)$, is the phase difference between the two rows, and whose magnitude, $M(n)=M_{A}(n) M_{B}(n)$, is the product of the magnitudes of each row. Now we use a simple least square distance estimator to obtain an estimate $\hat{P}$, over $L$ samples of the complex process. In other words, we need to find $\hat{P}$ so that the following cost function is minimum.

$$
C(\hat{P})=\sum_{n=1}^{L} W^{2} D^{2}(n)
$$

where $W$ is a window function and $D(n)$ is the distance between the estimate and the $n$th sample:

$$
\vec{D}(n)=\vec{P}(n)-\hat{P} .
$$

Solving (11) for $\hat{P}$ yields

$$
\hat{P}=\frac{\sum_{n=1}^{L} W^{2} \vec{P}(n)}{\sum_{n=1}^{L} W^{2}} .
$$

The above equation shows that the estimate of $L$ complex samples is the average of the corresponding $L$ samples.

This kind of estimate is also a maximum likelihood estimate, because: Process (10) at each time sample represents a complex number whose real and imaginary parts are sums of the combination products of the in-phase and quadrature samples of the signal and band-limited noise terms from arrays A and B [4]. Since the original noise that enters the quadrature receiver is a Gaussian noise due to ambient and sea reverberation, then for large SNR, we may ignore the noise-noise cross-product terms [4] and consider the noise terms in (10) as Gaussian zero mean noise. Thus the real and imaginary parts of (10) at each time sample are the corresponding original samples plus a zero mean Gaussian noise. On the other hand, since the seafloor slope changes are not very fast, we can consider the original data samples to be highly correlated within small time bins. Knowing these two facts, we can use a maximum likelihood estimator to estimate the real and imaginary parts of the data produced by processor (10) within each time bin containing $L$ samples. At this condition, the maximum likelihood estimate is the average of the corresponding measured data over the $L$ samples [6]. The result of this average is the same as (13). Thus, (13) is a maximum likelihood estimate of $L$ complex samples. 
Now, the estimated phase difference is the phase of $\hat{P}$ :

$$
\hat{\Delta} \phi=\tan ^{-1}\left\{\frac{\sum_{n=1}^{L} W^{2}(n) \operatorname{Im}\left\{S_{A}(n) S_{B}^{*}(n)\right\}}{\sum_{n=1}^{L} W^{2}(n) \operatorname{Re}\left\{S_{A}(n) S_{B}^{*}(n)\right\}}\right\}
$$

where $\operatorname{Re}\{\cdot\}$ and $\operatorname{Im}\{\cdot\}$ are the real and imaginary parts of $\{\cdot\}$. Equation (14) is an estimate of the phase of a complex sample whose real and imaginary parts are respectively the weighted mean of $L$ samples of the real and imaginary parts of the complex sequence (10). This phase difference estimation has the advantage of requiring only one $\tan ^{-1}$ operation for every $L$ samples, as opposed to one per sample as in (7). This prevents the injection of undesired phase differences due to the periodicity of the $\tan$ operation at $+\pi$ and $-\pi$.

The weighted mean processes in (14) are low-pass FIR filters with coefficients $W^{2}(n)$, acting on $L$ samples of the real and imaginary parts of the complex sequence $S_{A}(n) S_{B}^{*}(n)$.

\section{Estimation Uncertainty}

We use the variance of the distance to obtain an uncertainty figure for the phase difference estimation. From (10), (12), and (13) the variance of the distance $D(n)$ for every $L$ sample is:

$$
\hat{D}^{2}=\left(\hat{P}^{2}\right)-(\hat{P})^{2} \text {. }
$$

Where $(\hat{\imath})$ shows the expected or estimated value of $(\cdot)$ and

$$
\hat{P}^{2}=\frac{\sum_{n=1}^{L} W^{2} P^{2}(n)}{\sum_{n=1}^{L} W^{2}}=\frac{\sum_{n=1}^{L} W^{2}\left|S_{A}(n)\right|^{2}\left|S_{B}(n)\right|^{2}}{\sum_{n=1}^{L} W^{2}} .
$$

If the $D(\vec{n})$ has an isotropic distribution, then the estimation uncertainty of the phase difference, $\epsilon$, can be derived from Fig. 3(a) as:

$$
\tan ^{2} \epsilon=\frac{\hat{D}^{2}(n)}{(\hat{P})^{2}} .
$$

But, in general, $D(\vec{n})$ does not have an isotopic distribution. In this case we decompose the complex vectors $D(\vec{n})$ and $P(\vec{n})$, in two orthogonal components, one in the direction of the estimated vector $\hat{P}$ with the unit vector

$$
u=\frac{\hat{P}}{|\hat{P}|}
$$

and the other orthogonal to $\hat{P}$ with the unit vector $\vec{v}$, such that $\vec{u} \cdot \vec{v}=0$. Now, the variance of the distance $D(\vec{n})$ in (15) decomposes into two components as:

$$
\hat{D}_{u}^{2}=\hat{P}_{u}^{2}-(\hat{P})^{2}
$$

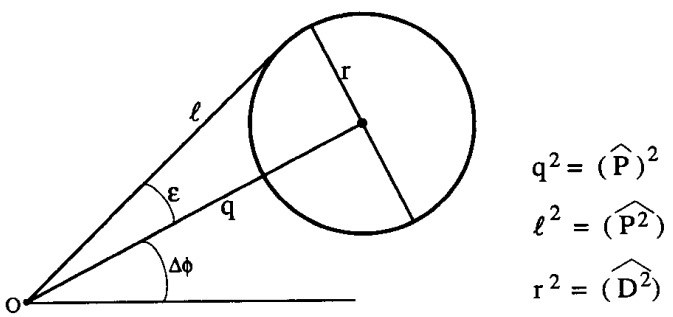

(a)

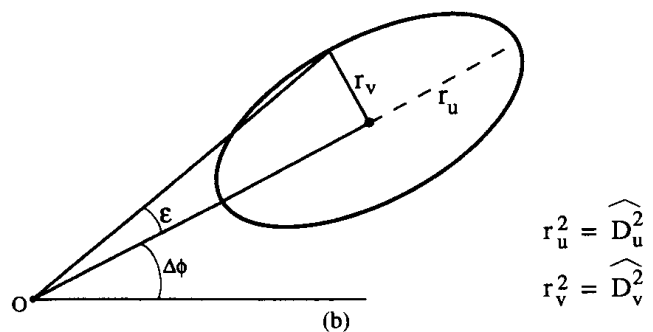

Fig. 3. Estimation uncertainty for (a) isotropic distribution (b) nonisotropic distribution.

and

$$
\hat{D}_{v}^{2}=\hat{P}_{v}^{2}=\hat{P}^{2}-\hat{P}_{u}^{2} .
$$

With this configuration the estimation uncertainty, $\epsilon$, can be obtained via Fig. 3(b) as:

$$
\tan ^{2} \epsilon=\frac{\hat{D}_{v}^{2}}{(\hat{P})^{2}}
$$

where

$$
\hat{D}_{v}^{2}=\frac{\sum_{n=1}^{L} W^{2} P_{v}^{2}(n)}{\sum_{n=1}^{L} W^{2}}=\frac{\sum_{n=0}^{L}\left[W^{2}(\vec{P}(n) \cdot \vec{v})^{2}\right]}{\sum_{n=1}^{L} W^{2}} .
$$

In practice the above estimation uncertainty is computed for the estimated phase difference sequence of a given ping, and the results are plotted versus sampling time for port and starboard sides.

\section{Application to SeamaRC II Data}

\section{A. System Characteristics}

The processing scheme outlined in Section II is applied to data recorded in the Norwegian-Greenland Sea in the Fall of 1990 with the SeaMARC II sonar system [5]. This is a long range swath bathymetric and imaging sonar designed to operate in a shallow tow mode (typically 100 $m$ below the surface) from ships of opportunity. Detailed descriptions of this sonar system are found in the literature [1], [2], so we only address the features relevant to the topic of this paper. The acoustic arrays consist of two rows of transducers per side. The arrays transmit a 1-2-ms pulse of $11 \mathrm{kHz}$ on port and $12 \mathrm{kHz}$ on starboard. For each side, both rows receive the bottom backscattered 
echoes. The corresponding signals are base-banded, lowpass filtered with a hardware filter whose nominal passband is $700 \mathrm{~Hz}$, and digitized at $4 \mathrm{kHz}$ per channel. These data are then processed by a real-time computer, according to the histogram method of phase processing mentioned above, and then converted to bathymetry for display on a printer. For the work described here, a special data acquisition system was set up to record the corresponding eight channels ( 2 pairs of quadrature components per side) on 9-track magnetic tapes for further processing. Keying on the transmit pulse, recording was set to start after a preset delay to account for the time it takes sound to travel to the bottom and back, and lasted 5-6 s, at which time a new transmit cycle was enabled.

The recorded data for a flat bottom region is processed by (10), and the phase difference, $\Delta \phi(n)$, and magnitude products, $M(n)$, are plotted for port and starboard sides in Fig. 4. The transmitted pulse in this example is $2 \mathrm{~ms}$. Similar plots are shown in Fig. 5 for another region that is not necessarily flat, and that is insonified with 1-ms pulse. The phase difference estimation starts after the first arrival sample and continues until the last sample.

The SeaMARC II system has several sources of noise and interferences that have been documented by Blackinton [2]. Two main sources of interference, inherent to almost all sidescan sonar systems, are the surface and bottom multiples. The surface multiple interference is due to the reflection of acoustic waves from the bottom to the surface and then to the towfish, or the opposite paths via the surface to the bottom and then to the towfish. The bottom multiple interference is caused by reflections from the bottom to the surface, back to the bottom and then received at the towfish. These interferences cause a rapid change in phase and magnitude of the recorded data at their starting point and spread out along the rest of the samples. In Fig. 4, the bottom multiple interference is clearly visible in the phase difference and magnitude plots, just before sample 14000 . In this example, the interference is so strong that the data cannot be recovered after the interference. Consequently, we truncate the process at the onset of the bottom multiple interference.

Before proceeding with the filtering process, we check the frequency content of the received signal for each row of the transducer array. Results of complex FFT processing applied to the data are shown in Fig. 6, for 1-ms and 2 -ms transmit pulses. FFT's are obtained on 1024 rectangular windowed samples (about $250 \mathrm{~ms}$ ) of the complex data for both port and starboard arrays $A$.

The power spectra exhibit the expected sinc function pattern for a rectangular pulse, as modified by the $700-\mathrm{Hz}$ passband of the analog receiver filter. The first nulls appear at about $500 \mathrm{~Hz}$ for the 2-ms pulse and about 1 $\mathrm{kHz}$ for the 1-ms pulse. However, in both cases, the spectra show a strong assymetry, with larger sidelobes on the right of the port spectra and on the left of the starboard spectra, indicating frequency leakage, or crosstalk, between the two sides whose center frequencies are only $1 \mathrm{kHz}$ apart.
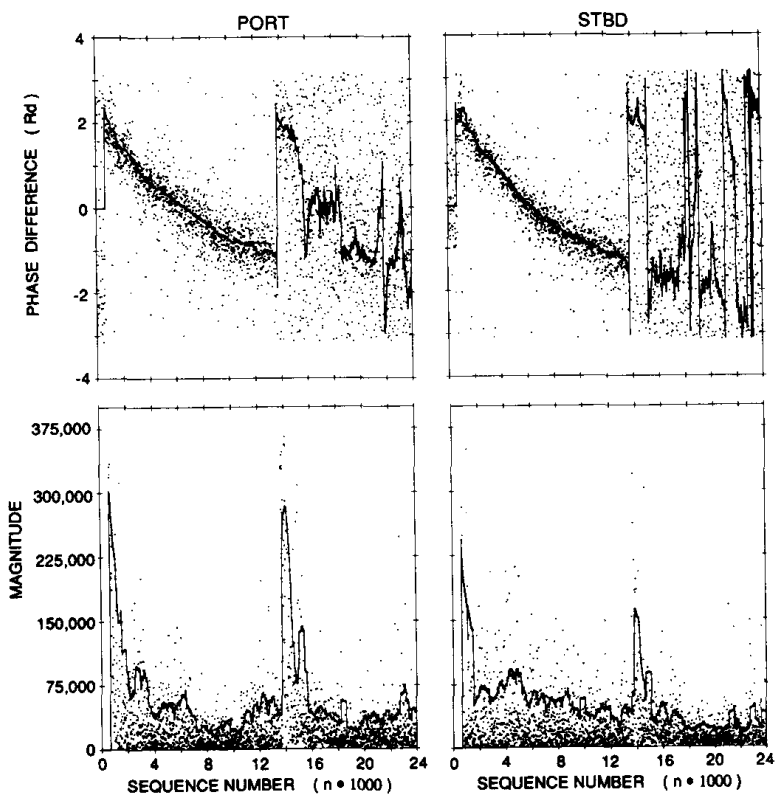

Fig. 4. Raw phase difference $\Delta \phi(n)$ and magnitude products $M(n)$ for echoes from a 2 -ms transmitted pulse. The solid line represents the corresponding results of the maximum likelihood estimation process. In this instance, the seafloor appears essentially flat at the resolution of the sonar. A bottom multiple is noticeable just before sample 14000 on both sides. A surface multiple appears around sample 1000 on the starboard side. Points in the sequence are $0.25 \mathrm{~ms}$ apart (4-kHz digitization).
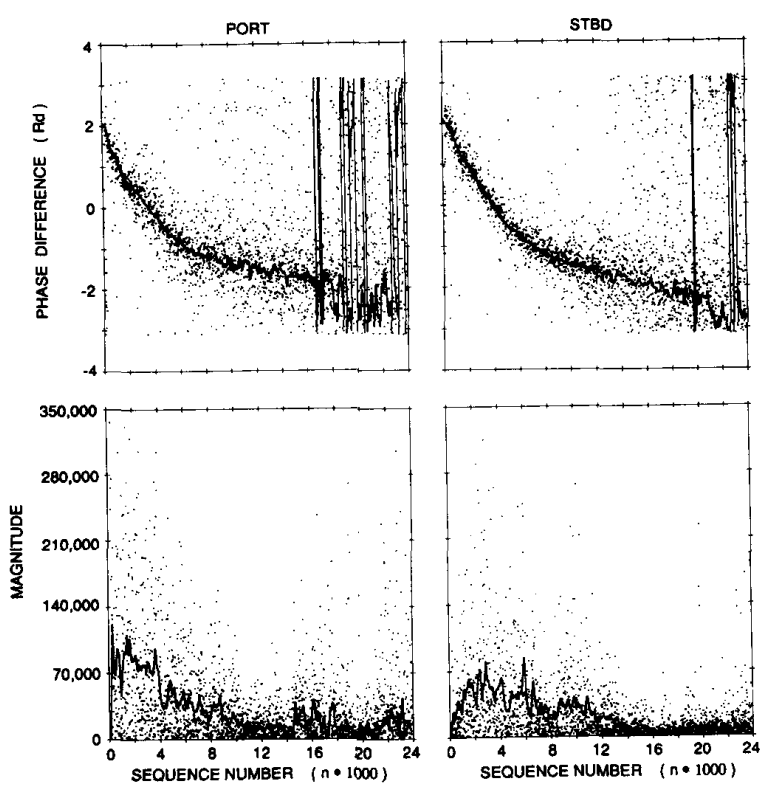

Fig. 5. Raw phase difference $\Delta \phi(n)$ and magnitude products $M(n)$ for echoes from a 1-ms transmitted pulse. The solid line is the output of the maximum likelihood estimator.

This crosstalk effect is emphasized in Fig. 7 with data collected while the transmitters were turned alternately on and off. In Fig. 7(a), with both sides transmitting a 

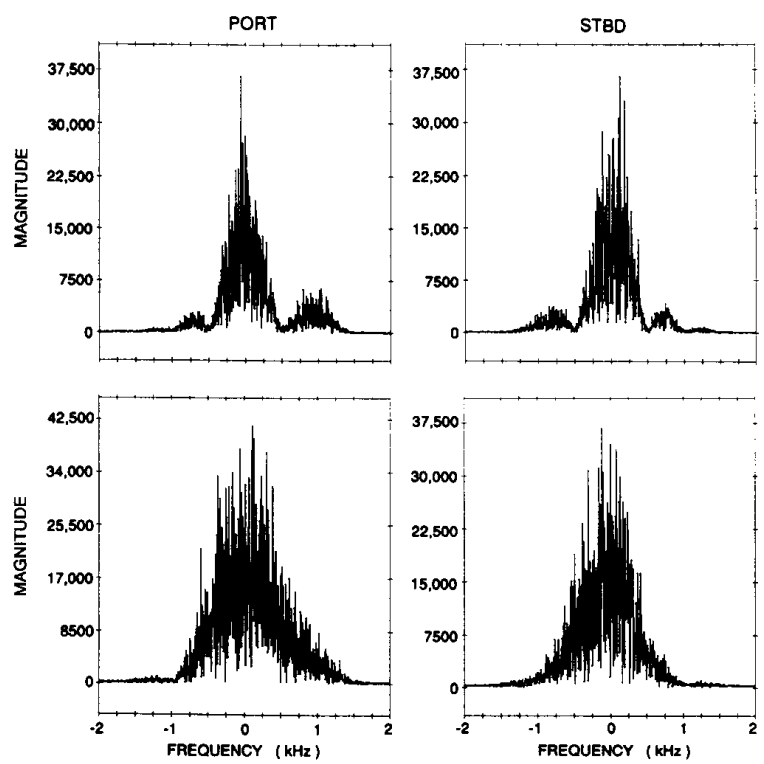

Fig. 6. Spectra of seafloor complex echoes received at row A on port and starboard, from transmitted pulses $2 \mathrm{~ms}$ (top) and $2 \mathrm{~ms}$ long (bottom).

2-ms pulse, the voltage spectrum of the echoes received on port array A shows the same assymetry as above. Transmitting with the port side only, results in a symmetric spectrum as seen in Fig. 7(b). Finally, transmitting with the starboard side only yields a spectrum of the echoes received on port as seen in Fig. 7(c). Here, only crosstalk is observed and its amplitude at $1 \mathrm{kHz}$ is only about $14 \mathrm{~dB}$ below the normal echo. Such in-band crosstalk would degrade the phase characteristics of a weak echo, especially when the seafloor on the opposite side is strongly reflective. Transmission of pulses $2 \mathrm{~ms}$ or longer would improve the phase measurement by narrowing the signal bandwidth, as would a better low-pass filter or a wider spacing between the port and starboard center frequencies.

In a new system, called HAWAII MR1, which operates in place of the SeaMARC II system lost at sea in February 1991, the same 1-kHz spacing between port and starboard has been retained to make use of existing components and speed up the new construction. However, a new all-digital signal processor makes it possible to implement a low-pass filter matched to the pulse width so as to reduce out-of-band interferences substantially. Here again, pulses shorter than $2 \mathrm{~ms}$ are almost never used.

\section{B. Differential Phase Estimation}

Next, we use the estimators (13) and (14) to extract the differential phase and the magnitude of bottom echoes. An FIR filter is used as the moving mean processor. The solid lines in Fig. 4 show the phase and magnitude estimated by (13), for a filter length of $L=400$ samples (100 $\mathrm{ms}$ ), using 2-ms pulse data, without prefiltering. As the filter length increases the filter frequency bandwidth decreases, yielding a smoother output. On the other hand,

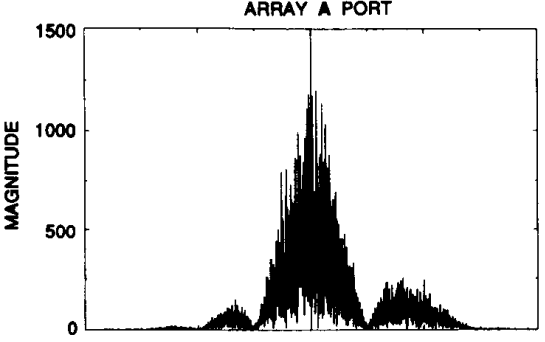

(a)

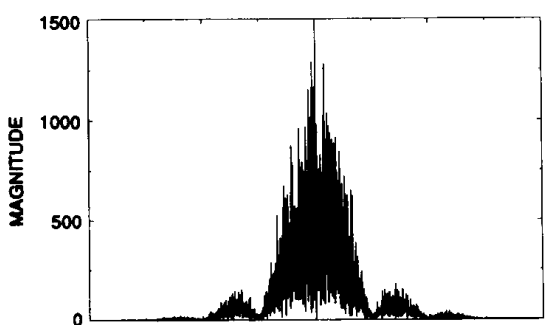

(b)

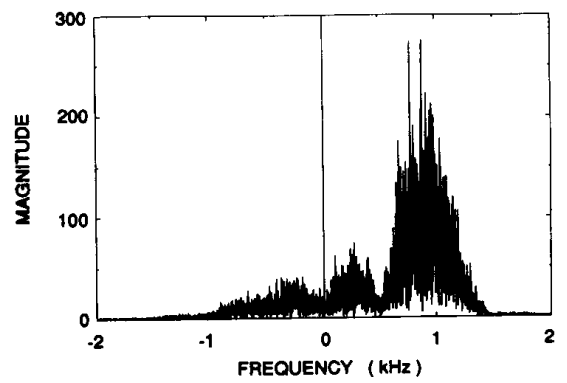

(c)

Fig. 7. Spectra of seafloor complex echoes received at row $\mathbf{A}$ on port, from a 2 -ms pulse transmitted from: (Fig. 7(a)) port and starboard simultaneously, (Fig. 7(b)) port only, and (Fig. 7(c)) starboard only.

increase in filter length causes loss in resolution and vice versa. Thus, it is a trade-off between smoothness and resolution, and one should take into account both factors in selecting the appropriate filter length. To evaluate this, a computer simulation is performed in Section IV. Here, the filter length is determined empirically by comparing the filter output with a reference data set obtained through ping stacking: the procedure will be explained in Section III.C, below. Then the estimation uncertainty for this filter length is obtained from (21) and the corresponding uncertainty in bearing angle can be computed for a flat bottom region. The uncertainty in bearing angle determines the resolution of the estimation process and it must be close to the theoretical resolution of the system, which is a function of the transmitted pulse length.

Figs. 8 and 9 show the estimation uncertainty for port and starboard. The large uncertainty values at the beginning are due to the transient effect. At the end they are due to the bottom multiple effect, which will be discussed later. The mean uncertainty in phase difference for the values in the middle range are 0.35 rad for port and 0.23 rad for starboard. The corresponding uncertainty in acoustic angle is found from the theoretical transfer func- 


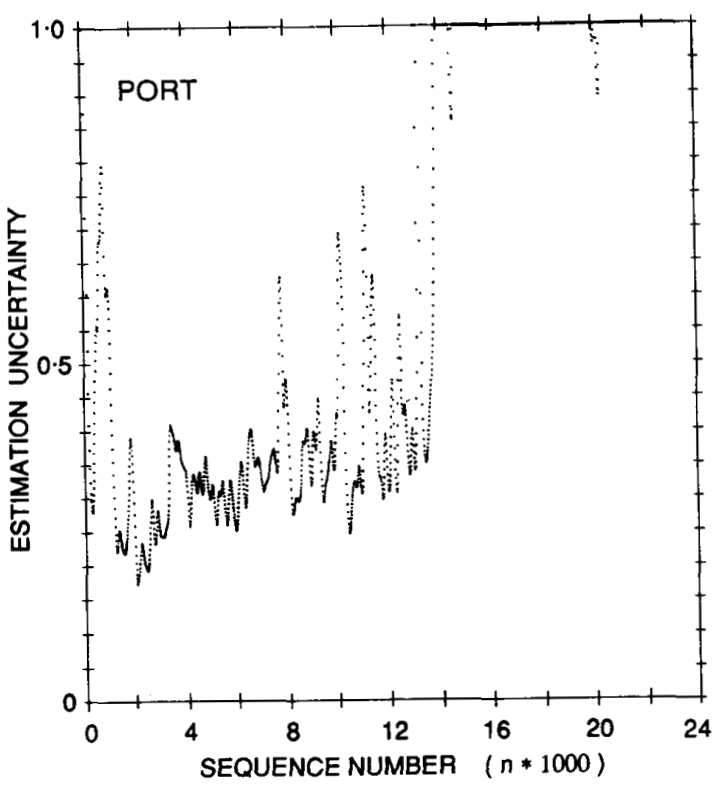

Fig. 8. Port estimation uncertainty.

tion (4) and is $6.5^{\circ}$ for port and $4.0^{\circ}$ for starboard. In practice (4) is not used as a transfer function between phase difference and acoustic angle, and an empirical table is used instead [1], [2]. Hence, the actual value of the estimation uncertainty in acoustic angle will be slightly different.

Given the smooth character of the estimated phase difference, only a subset of the original samples are needed to characterize it. To this end, the data are decimated to 128 samples through a median filter. Fig. 10(a) shows the decimated versions of the phase difference data (Fig. (4)). The above decimation is done between the first arrival and the bottom multiple samples. For comparison, Fig. 10(b) shows the results obtained on the same data set with the histogram procedure currently used in the SeaMARC II System. In this method, the phase difference $\Delta \phi(n)$ is binned in equi-spaced angles $\left(2.8^{\circ}\right)$ and the peak point of the histogram is selected as a modal time for every individual angle bin. Several instances of identical modal times for different angle bins are evident in Fig. 10(b). A modal angle picking algorithm would give slightly better results, but angle bins would be multivalued in time. These ambiguities require further processing to avoid artifacts in the corresponding bathymetry. This problem does not exist in Fig. 10(a). However, the smoothness of the phase curve resulting from filtering with a 100-ms FIR filter should not be compared to the output of the histogram method in which bins are only $23 \mathrm{~ms}$ wide.

\section{Evaluation of the Estimator}

In the following we consider data recorded when the sonar transmitted a 2-ms pulse, and compare the performance of the above estimation process with the uniform mean approach, which is known to be biased [1], [3].

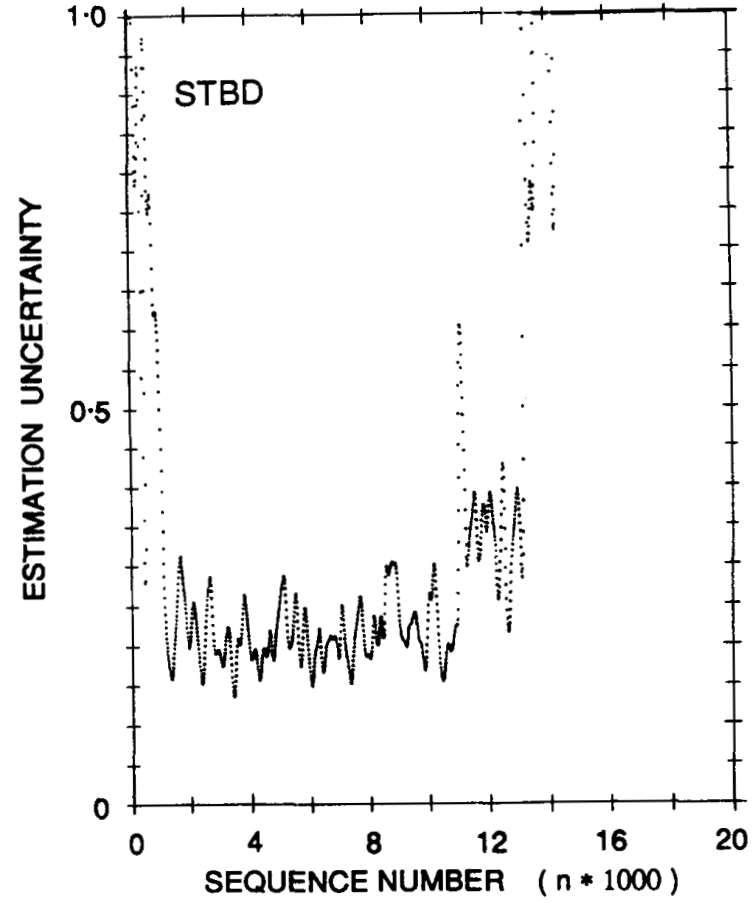

Fig. 9. Starboard estimation uncertainty.

According to estimation theory, an efficient estimator is one that is unbiased and has minimum variance [6]. The bias of the estimator is the expected value of the difference between the estimate of the parameter and its original value. The variance is the mean square error of the biased estimation. Thus, for this comparison we need a reference phase difference sequence that can be assumed to be the original value of the phase difference. Such a reference sequence was obtained, independently of the above procedure, by stacking and averaging each complex time sample for 115 contiguous pings recorded over a flat bottom region. As the area is flat, it is assumed that all the pings carry essentially the same bottom backscatter information with some noise. It is also reasonable to assume that the noise in each individual ping is a combination of ambient noise plus sea surface reverberation, that can be treated as independent zero mean Gaussian processes [4]. The uncorrelated noise sequences in different pings are cancelled significantly through this ping stacking process, and the resultant data are almost equivalent to a single "clean" data sequence. This estimate, which is equivalent to the maximum-likelihood estimate of the data, is unbiased, and its expected value must be equal to the original data sequence [6]. Results of the corresponding average phase differences for 115 pings with median filter decimation are illustrated in Fig. 11. To obtain an expected value of the stacked data closer to the original data would require more data recorded in the same fashion over the same area. For lack of such luxury, data in Fig. 11(a) represent the best reference sequence available. Also, this reference sequence has been kept as 

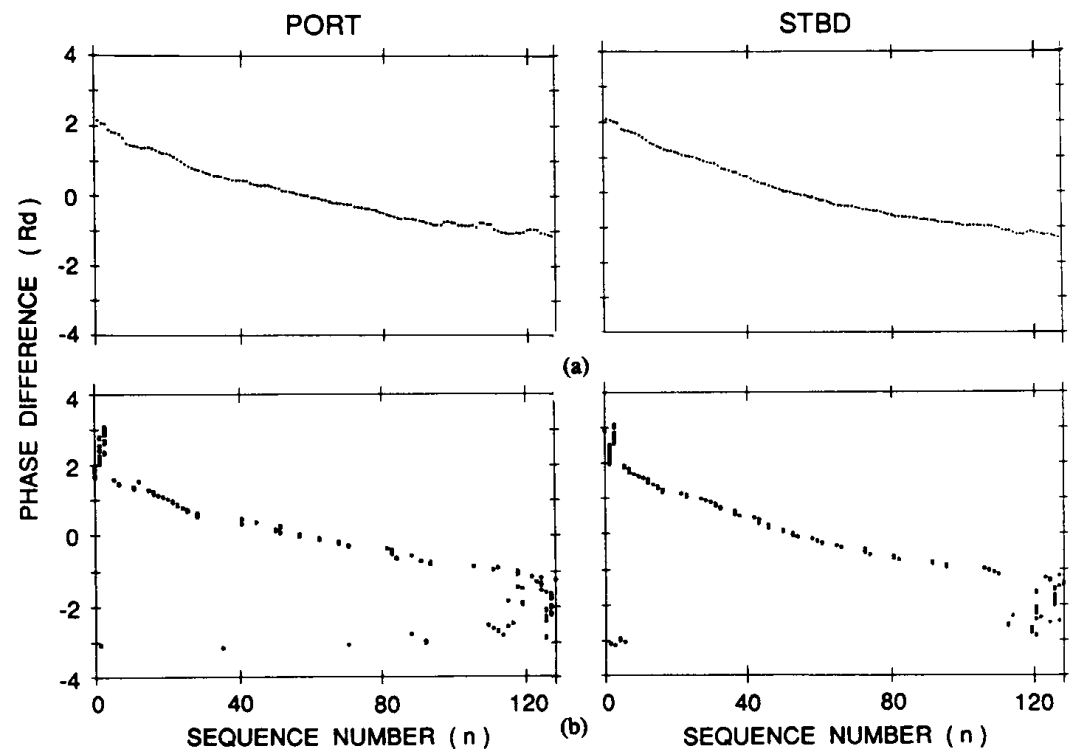

(a)

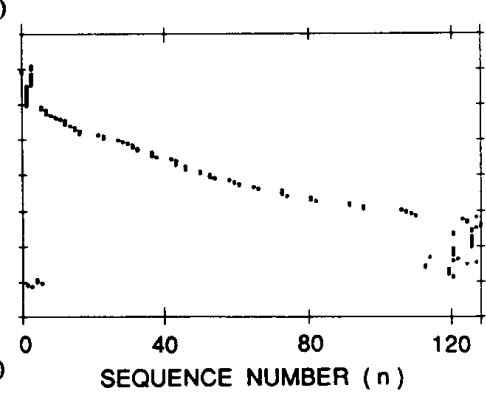

Fig. 10. (a) Decimated versions of phase difference obtained via the maximum likelihood estimator (14) using a rectangular window, and truncated between the first arrival and the first bottom multiple for the data shown in Fig. 4. (b) Phase difference estimation by the histogram method, using modal time picking, for the same data.
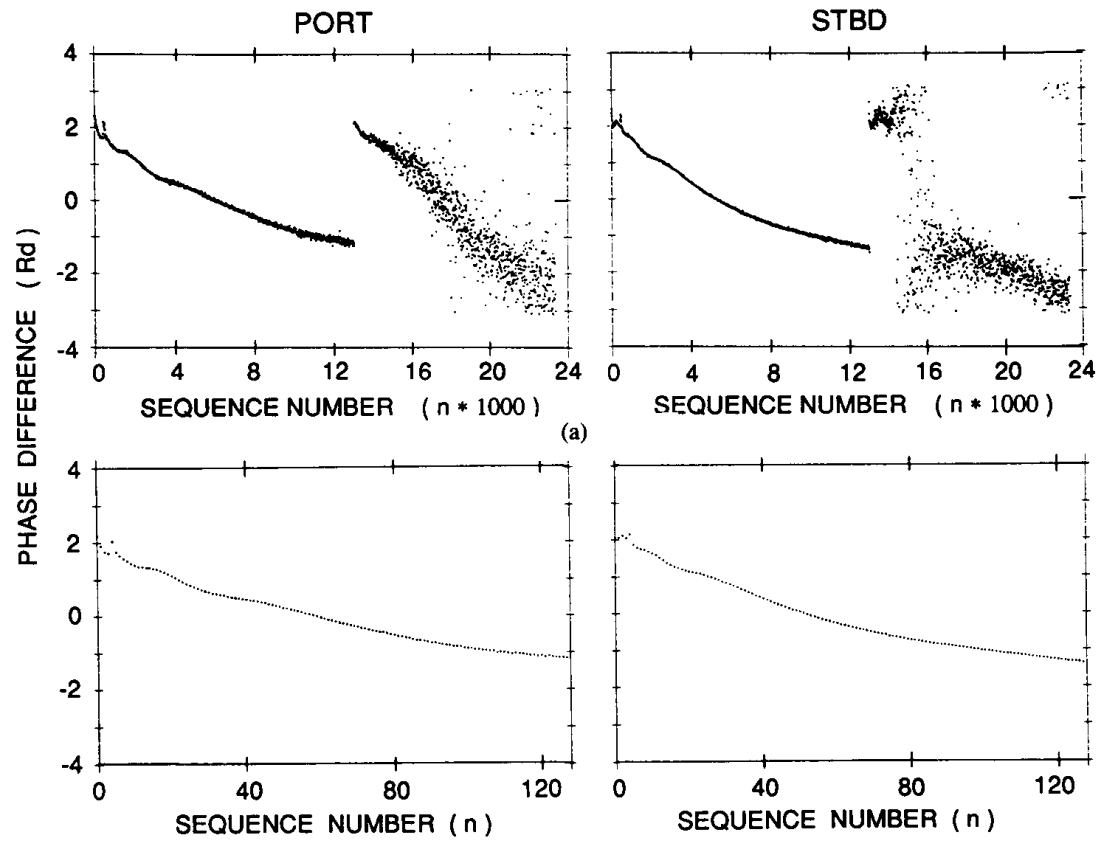

(a)

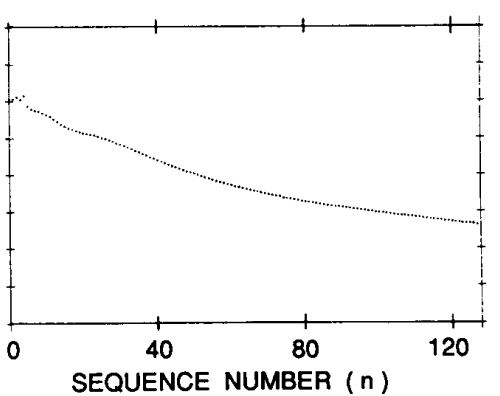

(b)

Fig. 11. Reference sequences (a) Average phase difference obtained by complex stacking of 115 contiguous pings recorded over a flat bottom area. (b) Median decimated version of (a), truncated between the first arrival and the first bottom multiple.

is, though it is somewhat noisy, because we know further filtering would potentially distort the "original" data characteristics.

Using this reference as the original data, we check the bias and variance of the maximum likelihood and uniform mean estimators. The bias is computed as follows: First, the differential phase is estimated either through the maximum likelihood estimation or the uniform mean for every individual ping; then, the expected value of this estimate is obtained by taking the mean over 115 pings. and eventually subtracting it from the original phase data (Fig. 11(a)) to yield the bias. Results of this algorithm are 

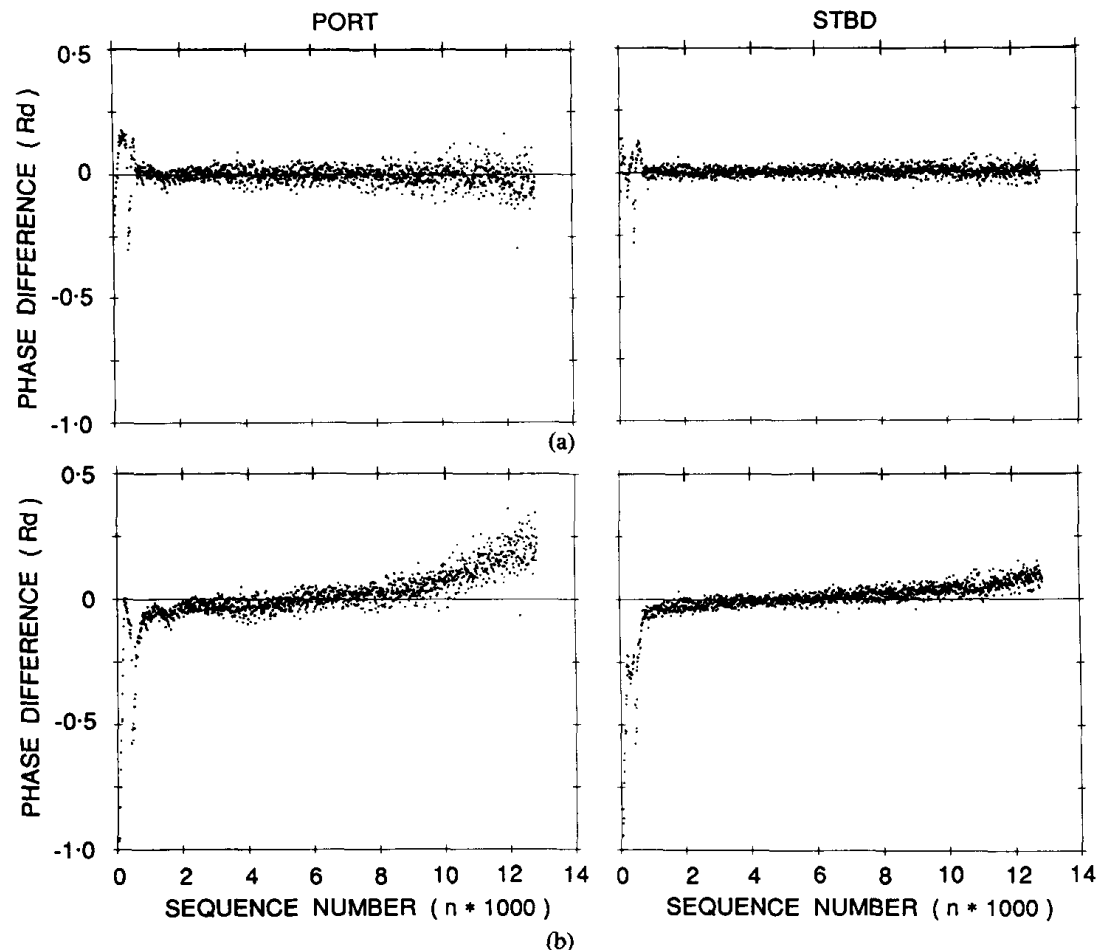

(b)

Fig. 12. Bias of the estimators: (a) maximum likelihood, (b) uniform mean. The bias is calculated on truncated sequences, and the offset from the origin around sample 500 in (a) and (b) corresponds to the surface multiple interference.

shown in Fig. 12(a) for the maximum likelihood estimate and $12(\mathrm{~b})$ for the uniform mean.

In graphical form, the amount of bias for any sample is represented by its deviation from the origin. The bias results in Fig. 12 indicate clearly that the maximum likelihood estimator has less bias than the uniform mean estimator, for both port and starboard sides. Deviations are larger in Fig. 12(b) than in Fig. 12(a), especially at the beginning and the end samples. The bias in these regions is mostly due to phase wrapping, which predominates at angles close to $\pi$ and $-\pi$ radians.

The other measure of performance of the estimator is the variance of the estimated phase difference with respect to the original phase difference data. We obtain the square of the error between the estimated value and the original value of the phase difference data for each individual ping, and find the mean of these values over 115 pings. The results from these computations are illustrated in Fig. 13(a), for the maximum likelihood estimate, and 13(b) for the uniform mean method. These figures also show that the corresponding variances for the maximum likelihood estimate are less than that of the uniform mean approach, which show the superiority of the maximum likelihood estimate over the other method. The large variance at the beginning and the end are due to the surface and bottom multiple interferences that cannot be treated by either method, although the above figures show that the maximum likelihood performance is better than the uniform mean for these interferences. Thus we con- clude that the maximum likelihood estimator shows better performance in estimating the phase difference.

\section{SEAMARC II ANALYTICAL MODEL AND COMPUTER SIMULATION}

So far we have been processing data recorded with SeaMARC II, and we have been limited to the parameters that have been set under the experimental environment. Here, we present an analytical model and computer simulation for the SeaMARC II system in order to have more flexibility in choosing the experimental parameters. This simulation also makes the processing and analysis of the data more reliable, controllable, and easier.

The model generates quadrature samples for arrays $\mathrm{A}$ and $B$ on either side for a flat bottom case. The analytical model is constructed on the basis of the reverberation process and its statistical characteristics that have already been established and addressed in the literature.

The reverberation process is considered as a quasiharmonic stochastic function which is given by (2) and (3) for rows A and B, respectively. We consider the following characteristics of the reverberation process in our model and simulation [7].

i) The quadrature components of a normal reverberation process are normal distribution processes with zero mean, and variances equal to the variance of the reverberation process.

ii) The quadrature components are uncorrelated and statistically independent. 

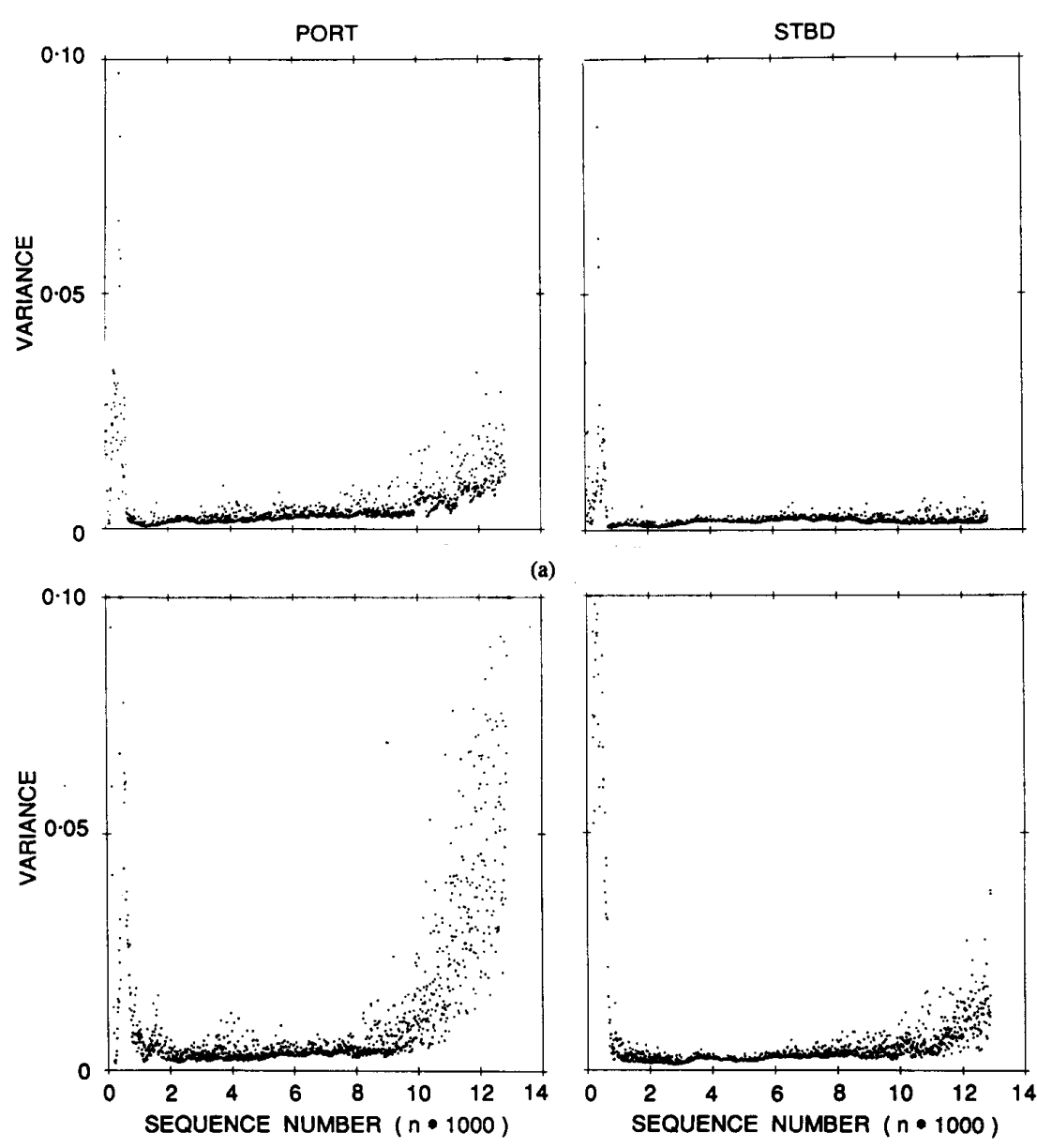

(a)

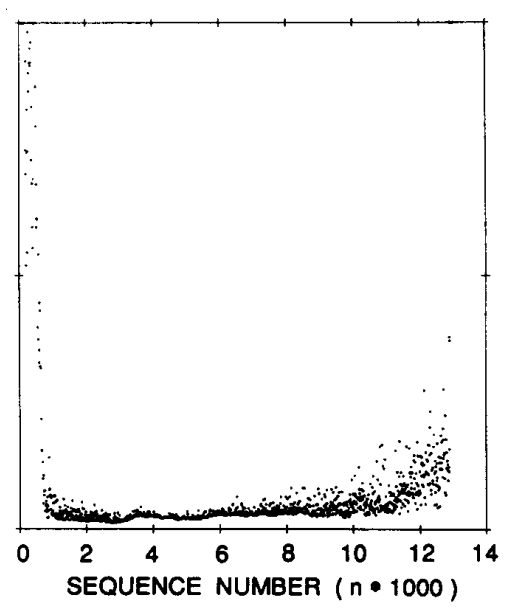

(b)

Fig. 13. Variance of the estimators: (a) maximum likelihood, (b) uniform mean. The variance is computed on truncated sequences. The surface multiple interference and the onset of the first bottom multiple lead to sharp increases in variance.

iii) If $S_{r}(t)$ is a stationary process, the phase $\phi(t)$ has uniform distribution in the interval $(0,2 \pi)$.

iv) The envelope of the reverberation process, $M(t)$, has a generalized Rayleigh distribution or Rice distribution.

v) The statistical spectrum of reverberation in the transmission of determinate-type signals (e.g., a gated pulse) is proportional to the square of their amplitude spectrum (e.g., a sinc function).

The model of the quadrature samples must contain all the above reverberation characteristics as well as the characteristics of the system, in both the time and frequency domains. In the time domain, the quadrature components of array $A$ are formed according to (5) and (6), with $\phi_{A}(n)$ randomly distributed in the interval $(-\pi, \pi)$ and $M_{A}(n)$ defined by two zero mean independent Gaussian processes $M_{1}$ and $M_{2}$ with variance $\sigma$ such that:

$$
M_{A}(n)^{2}=M_{1}(0 ; \sigma)^{2}+M_{2}(0 ; \sigma)^{2}
$$

in order to satisfy the conditions i) through iv) above. The spectral characteristics of the signal are obtained by convolving these quadrature components with a window function whose length is equal to the transmitted pulse length. This insures that condition v) above is satisfied because for stochastic processes in a linear system with transfer function magnitude $|H(\omega)|$, the spectral density of the output, $S_{Y Y}(\omega)$, and the input, $S_{X X}(\omega)$, are related by

$$
S_{Y Y}(\omega)=S_{X X}(\omega)|H(\omega)|^{2}
$$

The components of array B are then formed in a similar fashion with magnitude from Gaussian processes $M_{3}$ and $M_{4}$ :

$$
M_{B}(n)^{2}=M_{3}(0 ; \sigma)^{2}+M_{4}(0 ; \sigma)^{2}
$$

and phase derived from $\phi_{A}(n)$ above, (4), and a flat bottom at depth $D$

$$
\phi_{B}(n)=\phi_{A}(n)-\frac{2 \pi d}{\lambda} \sin \left(\theta_{m}-\theta(n)\right)
$$



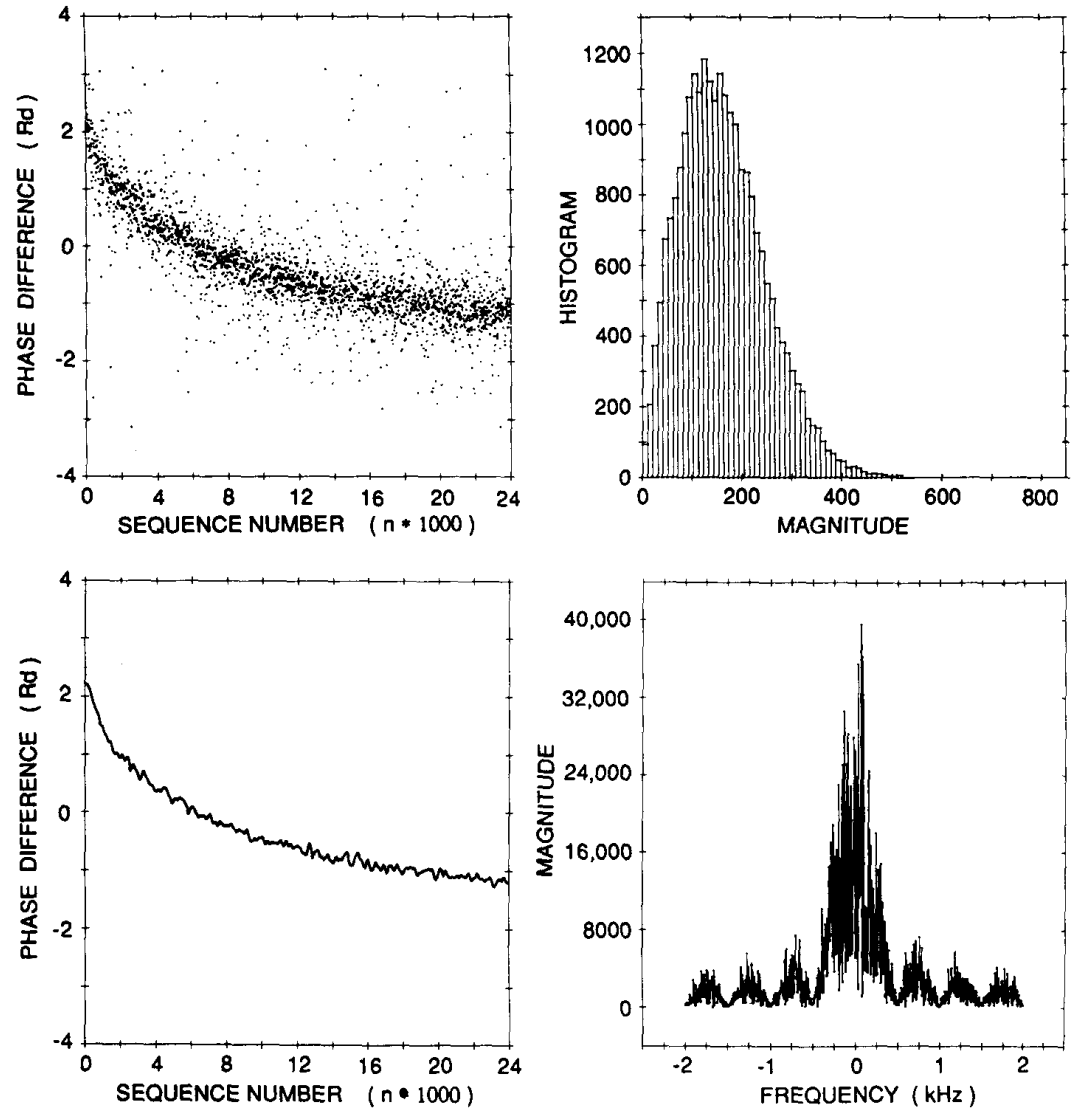

Fig. 14. Phase difference, estimated phase difference, histogram of magnitude of row A, and spectra of the simulation data for a 2 -ms pulse transmitted signal over a flat bottom region.

with

$$
\theta(n)=\cos ^{-1}\left(\frac{2 D}{c n}\right) .
$$

Uncorrelated band-limited, zero mean noise processes with variance $\sigma_{0}$ are also added to the quadrature component $I_{B}$ and $Q_{B}$ to simulate ambient noise contributions.

This model is meant to verify the signal processing scheme employed here. It does not address the physical parameters related to propagation of sound waves in the water column or their interactions with the bottom. Only the geometry of the bottom is taken into account. Here we have simulated a flat horizontal plane at about $2400 \mathrm{~m}$ depth.

Fig. 14 shows the model raw phase difference, the histogram of the magnitude, the estimated phase difference, and the spectrum for a 2-ms pulse length. In this process we have considered $\sigma=1000$ and $\sigma_{0}=400$. We observe that all the simulation results compare quite closely to the corresponding real data results. The shape of the power spectrum reflects the ideal rectangular nature of the window used to simulate the transmitted pulse. It does not include the taper characteristic of a real transmitted pulse, nor the "smoothing and smearing" effects resulting from the $700-\mathrm{Hz}$ low-pass hardware filter found in the SeaMARC II receivers. The corresponding estimation uncertainties for $50-\mathrm{ms}, 100-\mathrm{ms}$, and $200-\mathrm{ms}$ filter lengths ( 200,400 , and 800 points, respectively) are in Fig. 15. Results for a 1-ms pulse are shown in Fig. 16.

These plots indicate that the estimation uncertainty is always lower for a 2-ms pulse. This can be explained by the fact that the narrower bandwidth signal carries less noise power with the quadrature samples. Moreover, the estimation uncertainty analysis is based on a pure tone assumption

for the backscatter signal, so that the narrower the bandwidth, the more accurate is the estimation uncertainty, i.e., smaller $\epsilon$.

Comparing the estimation uncertainty results for different filter lengths, in Figs. 15 and 16, we observe that the initial mean value increases with the filter length whereas the variance decreases. This shows that filter lengths must be small at the beginning of the sequence and large at the end. In the work described above using actual SeaMARC II data, we compromise by choosing an intermediate value for the filter length and keeping it constant through the entire sequence.

\section{CONCLUSION}

A processing scheme based on the maximum likelihood (least square distance) approach has been introduced to 

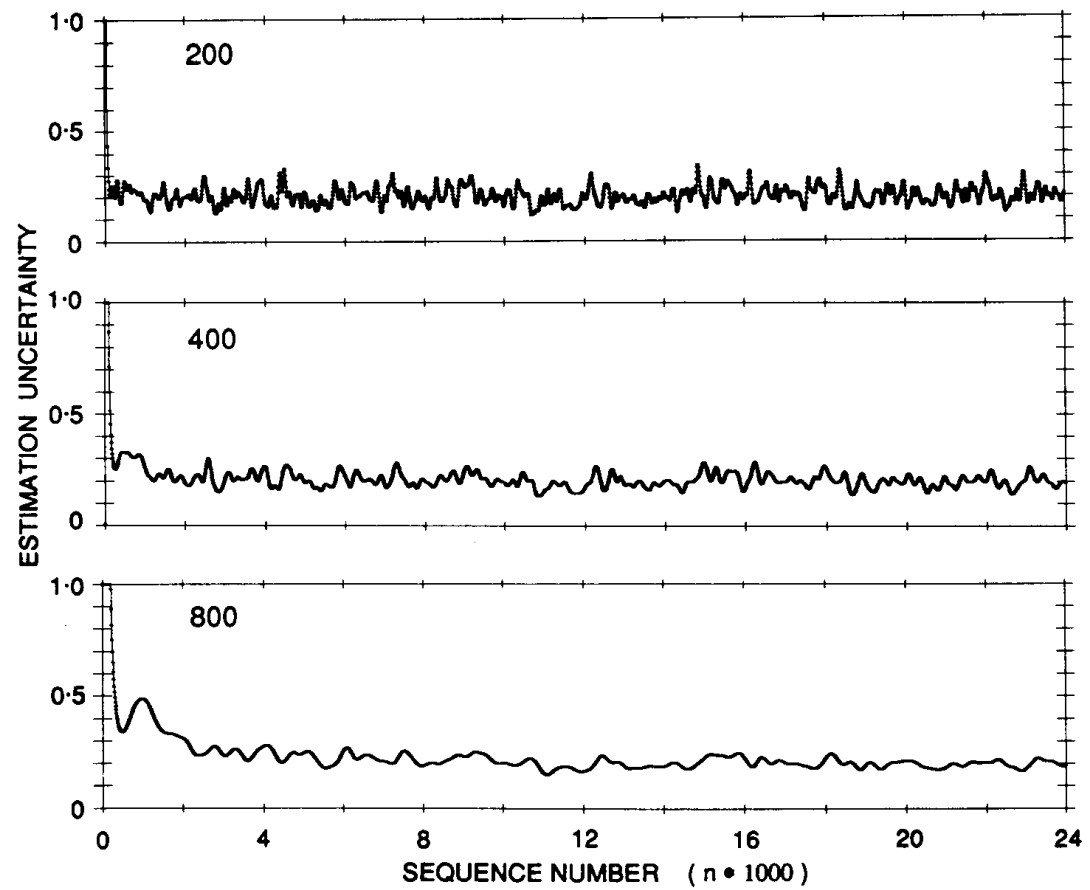

Fig. 15. Estimation uncertainty for the simulation data for a 2 -ms pulse over a flat bottom region with 200 , 400 , and 800 sample filter lengths.

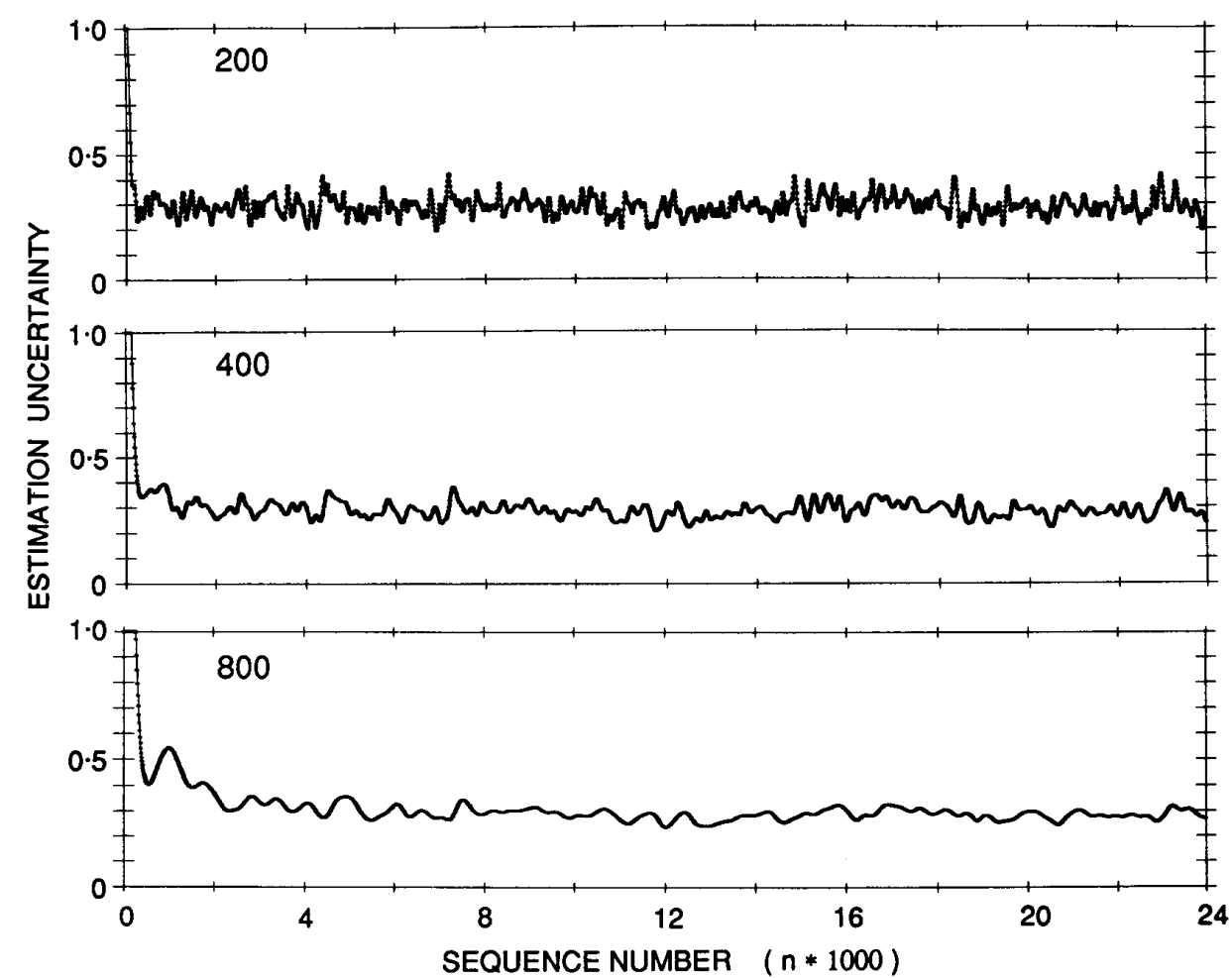

Fig. 16. Estimation uncertainty for the simulation data for a 1 -ms pulse over a flat bottom region with 200 , 400 , and 800 sample filter lengths. 
estimate the phase difference of noisy seafloor echoes received by pairs of transducer rows on the SeaMARC II bathymetric sidescan sonar system. This estimator works on the full complex digital sequence recorded from each array.

The estimation was evaluated with data recorded at sea and its performance was compared to that of a uniform mean method and to the histogram method currently used in the system. To determine the bias and the variance of the estimator, a reference differential phase sequence was obtained by complex stacking and averaging several contiguous pings recorded over a flat seafloor region. The new estimator was shown to be more efficient, i.e., with less bias and smaller variance, than the uniform mean estimator. The results are also superior to those obtained via the histogram method, because they do not suffer from ambiguities caused by dispersed phase values yielding different phase angles for common arrival times or different arrival times for a given phase angle. Ambiguities resulting from multiple targets at the same angle by different ranges, or at the same range but different angles cannot be resolved by any of these methods.

The resolution of the system was determined by an evaluation figure called "estimation uncertainty." An analytical modeling and computer simulation of the system was also presented and all the real data processing results were evaluated in the simulation. Simulation results confirmed that the estimation uncertainty decreases with decreasing signal bandwidth.

\section{ACKNOWLEDGMENT}

The authors are indebted to a number of people who made the data collection at sea possible: $M$. Rognstad for the hardware and R. M. Lawhead for software contributions; the chief scientists of the expedition, K. Crane, E. Sundvor, and P. Vogt, and the scientific party and the crew of the R. V. Haakon Mosby, operated by the University of Bergen, Norway. This work has benefited from discussions on the phase measurement problem with $\mathrm{H}$. Matsumoto and J. Krolik.

\section{REFERENCES}

[1] H. Matsumoto, "Characteristics of SeaMARC II phase data," IEEE J. Oceanic Eng., vol. 15, pp. 350-360, 1990.

[2] J. G. Blackinton, "Bathymetric mapping with SeaMARC II: An elevation angle measuring side-scan sonar system," Ph.D. dissertation, Hawaii Inst. Geophys., Univ. Hawaii, Manoa, 1986.

[3] M. Shensa and C. Black, "Passive bearing estimation: The removal of bias and $2 \pi$ ambiguities," J. Acoust. Soc. Amer., vol. 63, pp. $91-100,1978$

[4] W. S. Burdic, Undenwater Acoustic System Analysis. Englewood Cliffs, Prentice Hall, 1991.

[5] E. Sundvor, P. R. Yogt, and K Crane, "Preliminary results from SeaMARC II investigations in the Norwegian-Greenland Sea," Seismo Series No. 48, Inst. Solid Earth Physics, Bergen, Norway, 1990.

[6] J. L. Melsa and D. L. Cohn, Decision and Estimation Theory. New York: McGraw-Hill, 1978

[7] V. V. Ol'sheveskii, "Characteristics of sea reverberation," Consultants Bureau, New York, 1967.

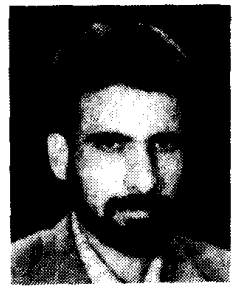

Mohammad A. Masnadi-Shirazi (M'90) received the B.S. and M.S. degrees in electrical engineering from Shiraz University, Shiraz, Iran, in 1974 and 1984, respectively, and the Ph. D. degree in electrical and computer engineering from the University of New Mexico. Albuquerque, in 1990.

He was teaching at Shiraz College of Electronics from 1974 to 1984 , and a teaching assistant in the Department of Electrical and Computer Engineering at the University of New Mexico from 1986 to 1990 . He was a postdoctral researcher at Scripps Institution of Oceanography, University of California, San Diego, from 1990 to 1992 . In 1992 he joined the Department of Flectrical Engineering at Shiraz University, where he is now an Assistant Professor. His research interests are in the area of systems and signal processing, pattern recognition, digital image processing with applications to sonar and radar data, and statistical communications.

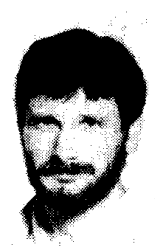

Christian de Moustier (M'86) received the Diploma of Engineer from the Ecole Supérieure d'Ingénjeurs de Marseille (France), in 1979, the M.S. and Ph.D. degrees in Oceanography (Applied Ocean Science) at the University of California, San Diego in 1981 and 1985, respectively.

$\mathrm{He}$ works at the Scripps Institution of Oceanography as an Assistant Research Oceanographer at the Marine Physical Laboratory, and as an Academic Administrator for Ship Operations and Marine Technical Support at Scripps. His present research interests are in underwater acoustics with emphasis on physics of bottom-interacting sound and sound reverberation in the ocean, seafloor acoustic imaging and contour mapping, and digital image processing applied to sonar data.

He was Guest editor for the October 1989 special issue of the Journal of Oceanic Engineering, devoted to bathymetry and seafloor acoustic remote sensing and presently serves as Associate Editor for these topics. $\mathrm{He}$ is a member of the Acoustical Society of America and the American Geophysical Union.

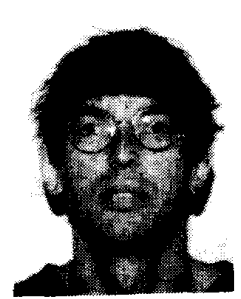

Pierre Cervenka received the doctorate degree in acoustics (1982) and in physics (1988), both from the Université Pierre et Marie Curie (Paris $6)$.

In 1983, he joined the Centre National de la Recherche Scientifique (CNRS) as a research scientist attached to the Laboratoire de Mécanique Physique et Expérimentale (Paris 6). Most of his studies were involved with nonlinear acoustics and modelization of parametric antennae. Since 1989, he has been a visiting assistant researcher at the Marine Physical Laboratory of the Scripps Institution of Oceanography, where he initially came under a CNRS-NSF exchange program.

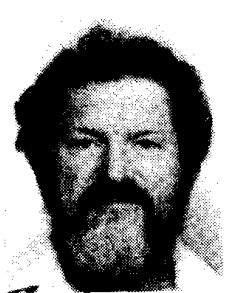

Stanley H. Zisk received the B.S. and M.S. degrees from MIT in 1953 and the Ph.D. from Stanford in 1965, all in electrical engineering.

He worked at MIT Haystack Observatory as a member of the Research Staff for a number of years on a combination of basic research in radio and radar astronomy, and development of cryogenics and maser amplifier microwave instrumentation, before succumbing to New Eng. land winter weather and moving to the University of Hawaii Planetary Geosciences group in 1988 as a Planetary Scientist. He has been working with radar and sonar image experiments and research, lately in collaboration with the HIG SeaMARC II Group (now the Hawaii Mapping Research Group), since that time. His current interests are underwater acoustics and the noise and structural characterization of acoustic geological images. 